PHYSICAL REVIEW D 89, 084009 (2014)

\title{
Intrinsic angular momentum for radiating spacetimes which agrees with the Komar integral in the axisymmetric case
}

\author{
Emanuel Gallo and Osvaldo M. Moreschi \\ FaMAF, Universidad Nacional de Córdoba Instituto de Física Enrique Gaviola (IFEG), \\ CONICET Ciudad Universitaria, 5000 Córdoba, Argentina
}

(Received 27 August 2013; published 2 April 2014)

\begin{abstract}
Here, we present a new definition of intrinsic angular momentum at future null infinity, based on the charge-integral approach. This definition is suitable for the general case of radiating spacetimes without symmetries, which does not suffer from supertranslation ambiguities. In the case of axial symmetry, this new definition agrees with the Komar integral.
\end{abstract}

DOI: 10.1103/PhysRevD.89.084009

PACS numbers: 04.25.dg

\section{INTRODUCTION}

The subject of physical quantities, such as momentum and intrinsic angular momentum, is always related to the notion of symmetries of the physical system to which they refer. Thus, in the framework of special relativity, we have at our disposal the definitions of total momentum and total angular momentum, based on the existence of the 10 Killing symmetries of the spacetime. When considering the analogous situation for an isolated system, in the framework of general relativity things are more complicated. One is faced with the difficulty that, in the neighborhood of future null infinity $\left(\mathcal{I}^{+}\right)$, the asymptotic symmetries generate an infinite dimensional group, namely, the Bondi-Metzner-Sachs (BMS) [1-3] one. This is because the existence of gravitational radiation affects the curvature of the spacetime, even in the asymptotic regime.

In the study of asymptotically flat spacetimes, it is tempting to express the asymptotic structure in terms of a decomposition of the metric over a flat one, namely,

$$
g_{a b}=\eta_{a b}+h_{a b},
$$

where $\eta_{a b}$ is a flat metric and $h_{a b}$ the tensor in which all the physical information is encoded. But the problem is that there are as many flat metrics as there are proper BMS [2,4] supertranslation generators. We should probably note that this difficulty is rooted in the existence of gravitational radiation, which reaches future null infinity, where total physical quantities are calculated. On the contrary, in the case of a stationary system, which therefore has no gravitational radiation content, one can single out a unique flat background metric $\eta_{a b}$, which one can use in (1).

The situation today is that there are numerous references for definitions of angular momentum at future null infinity, for general radiating spacetime; most of them suffer from the so-called problem of supertranslation ambiguities [5-9], but the physics community has not yet embraced a standard for it (for a review and references on the subject of energymomentum and angular momentum in general relativity, see [10]). This is in spite of the fact that in [11] a definition of intrinsic angular momentum was presented, free from supertranslation ambiguities, satisfying a set of appropriate physical conditions. However, in this reference, it was not resolved the relation between that definition and the Komar integral in the case of the existence of an axial symmetry. Although the presence of a rotational Killing vector is not the usual situation, its study gives important clues on invariant definitions of angular momentum. Since, under these circumstances, the Komar integral becomes an important tool due to its conservation properties, in this article we tackle this problem by presenting a new definition of intrinsic angular momentum, free from supertranslation ambiguities, which agrees with the Komar integral in the particular situation of the existence of axial symmetry. Therefore, the main improvement of this work over [11], which has already provided a definition of intrinsic angular momentum free of supertranslation ambiguities, is to answer our question [11] and other criticisms [12] about the relation of this approach to the Komar integral.

It is probably worthwhile to remark that most of the numerous definitions of angular momentum at future null infinity do not tackle the problem of intrinsic quantities.

In our work we will use extensively a definition of rest frames [13-15], which is described below, along with its relation to the definitions of center of mass and intrinsic angular momentum. Using these definitions we can further select a unique timelike orderly family of sections that have information for the center of mass and intrinsic angular momentum [11].

Therefore, for each point at future null infinity, we have [11] a way to single out a unique decomposition of the metric in the form (1), with an appropriately selected flat background $\eta$.

In order to gain perspective of our work let us consider a radiating asymptotically flat spacetime in which one can distinguish three stages. The first stage is when the asymptotic region possesses an axial symmetry, the second stage is when no symmetry is found, and the third stage is when one finds another axial symmetry, which does not coincide with that of the first stage. The situation 
is depicted in Fig. 1. Here, we present new definitions of center of mass and intrinsic angular momentum which are suitable for the general case of radiating spacetimes, that do not suffer from supertranslation ambiguities, and which in the first and third stages, where two different rotational Killing vectors exist, coincide with the Komar integral. To our knowledge, these are the only definitions of center of mass and intrinsic angular momentum which possess these properties. It is important to emphasize that the corresponding center of mass in the first and third stages would, in general, involve a supertranslation (see Fig. 2).

Our work is based on the charge-integral approach. In the past, Penrose [16] used the notion of charge integrals of the

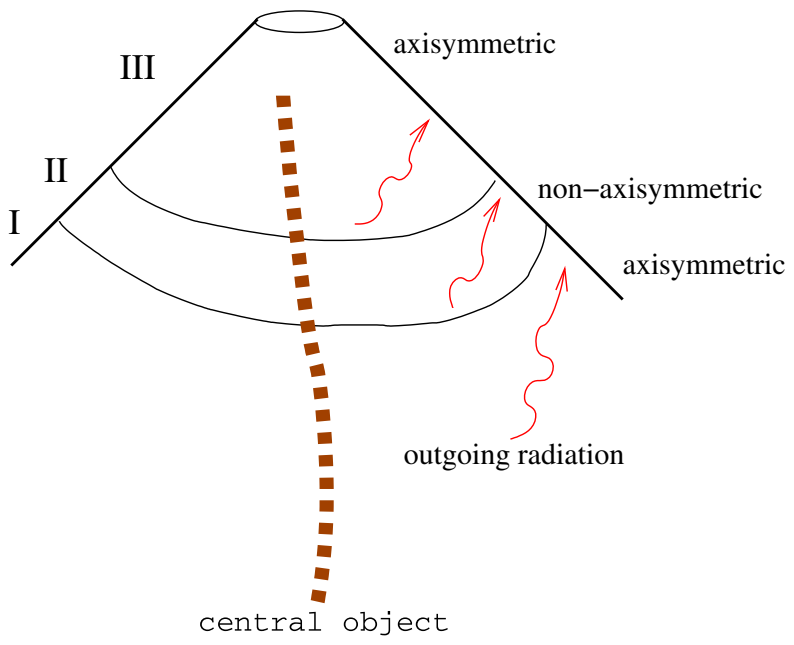

FIG. 1 (color online). A spacetime whose asymptotic regions have three different stages.

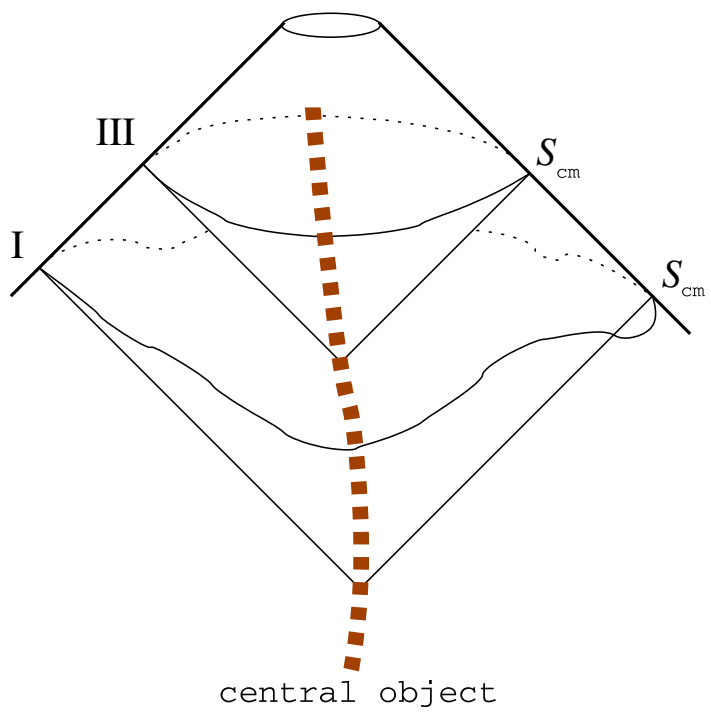

FIG. 2 (color online). Any intrinsic definition of center of mass will necessarily involve supertranslation in different instances of the dynamical system.
Riemann tensor in his study of quasilocal mass and angular momentum. We have used the same approach in our work on total angular momentum [4], with a global choice of reference frame. Later, in [11], we used this concept in our construction of a definition of intrinsic angular momentum free of supertranslation ambiguities. One advantage of the approach of charge integrals is that for each generator of asymptotic symmetry, one has an associated physical quantity; in particular, the same expression provides a definition not only of angular momentum but also of total linear momentum. Furthermore, by construction, the factor of 2 anomaly problem, found in the Komar integrals [7], is absent in this formalism.

We are presenting, for the first time, a definition of intrinsic angular momentum in general relativity which is free from supertranslation ambiguities, valid for the most general class of isolated systems, and it agrees with the Komar integral in the presence of rotational Killing vectors. We think that this work will be of interest to a variety of readers; some of them might not be experts in the subject, but the definition might be relevant for their work. For this reason we try to present it in a form as self-contained as possible, so some expert readers are advised to overlook sections with standard definitions.

The paper is organized as follows. In Sec. II, we present a brief review of the concepts of rest frames, supermomentum and nice sections, information that readers already acquainted with these notions may omit. In Sec. III, the Komar integral is expressed in terms of spinorial quantities using the Geroch-Held-Penrose (GHP) [17] formalism. In Secs. IV and V, we discuss the charge-integral approach, and finally, in the last section, we present the new construction and definition of intrinsic angular momentum valid for general asymptotically flat spacetimes.

\section{II. "NICE SECTIONS" AS A TOOL FOR THE SUPERTRANSLATION PROBLEM}

\section{A. Too many rest frames}

As was mentioned in the Introduction, in spacetimes which are asymptotically flat at future null infinity, instead of the Poincare group with a finite number of symmetries, we have the BMS group with an infinite number of asymptotic symmetries. Although this group contains the subgroup of translations as a normal subgroup (which allows us to define geometrically the linear total Bondi momentum), it does not contain a subgroup of Lorentz rotations defined in a canonical way. Therefore, there is no a priori intrinsic way to define a Pauli-Lubanski-like vector. To see some specific examples where these constructions would fail, we refer to the work [18], where it is shown, in particular, that a supertranslated boosted section in Schwarzschild spacetime would give a nonzero angular momentum, which, if used in a Pauli-Lubanski-like vector 
[19], would produce a quantity that is supertranslation dependent. However, we can base the analysis in terms of the supermomentum, which is defined in terms of the infinite supertranslation generators and which we use to define the concept of nice sections. The supermomentum is an object with infinite components such that the first four of them define the total Bondi momentum. Using this concept, we can ask whether there are sections at $\mathcal{I}^{+}$such that on these sections the only nonvanishing component of the supermomentum is the first one, that is, the timelike component of the Bondi momentum. This would allow us to give a definition of rest frames that we materialize in the concept of nice sections. But for this program to really work, one should prove, among other things, that this family of sections is actually a four-parameter family. Fortunately, it was proven in the past [13-15] that this program can be successfully carried out. With the concept of nice sections at hand, we have at our disposal a tool to single out a Lorentz subgroup of the BMS group for each rest frame. We will now review some of these concepts.

\section{B. Bondi systems as inertial frames}

The analogues to inertial frames of special relativity at future null infinity are the Bondi systems, by which we mean [4] a coordinate and tetrad system, which we describe below. Let $\left(M, g_{a b}\right)$ be an asymptotically flat spacetime at null infinity [20]. As it was mentioned in the introduction, in a vicinity of $\mathcal{I}^{+}$one can express the metric in terms of a flat metric $\eta$ plus a tensor $h$; where $h$ goes to zero appropriately [21] as one approaches future null infinity. Each Bondi system can be used to build such a flat metric $\eta$, and any other Bondi system connected to the first by a translation or a Lorentz rotation will determine the same flat metric $\eta$. However, any other Bondi system connected to the first by a proper supertranslation will determine a different flat metric tensor $\eta$. This situation is what complicates the discussion of global quantities such as angular momentum at $\mathcal{I}^{+}$.

Let us explain what we mean by Bondi systems. In the vicinity of $\mathcal{I}^{+}$, we can construct a coordinate system $(u, r, \zeta, \bar{\zeta})$, where $u$ are null hypersurfaces, $r$ is an affine parameter of the null generators $l^{a}=g^{a b}(d u)_{b}$ of the null hypersurfaces $u=$ const (such that when $r$ goes to infinity, the integral curves of these null generators intersect $\mathcal{I}^{+}$), and $(\zeta, \bar{\zeta})$ are stereographic coordinates labeling the null generators of $\mathcal{I}^{+}$. If this coordinate system is chosen such that when $r$ goes to infinity the induced (conformal) intrinsic metric on $\mathcal{I}^{+} \hat{g}_{a b}=\Omega^{2} g_{a b}$ with $\Omega=r^{-1}$ is the standard metric of a unit sphere, or more precisely, if the metric $\left.\hat{g}\right|_{I}$ on $\mathcal{I}^{+}$reads

$$
\left.\hat{g}\right|_{I}=0 \cdot d u^{2}-4 \frac{d \zeta d \bar{\zeta}}{(1+\zeta \bar{\zeta})^{2}},
$$

then the coordinate system $(u, \zeta, \bar{\zeta})$ defines a Bondi system. One can further require the affine coordinate $r$ to agree with the so-called "luminosity distance" [21] so that the coordinate system has an invariant extension into the interior of the spacetime. Associated to this coordinate system, we have a null tetrad $\left\{l^{a}, n^{a}, m^{a}, \bar{m}^{a}\right\}$, in the vicinity of $\mathcal{I}^{+}$, where $n^{a}$ is a null vector such that $l^{a} n_{a}=1$, with $m^{a} \bar{m}_{a}=$ -1 and all other possible products vanishing; $m^{a}, \bar{m}^{a}$ are complex null vectors tangent to the two-spheres defined by $u=$ const and $r=$ const.

While inertial frames of special relativity are related by Poincaré transformations, Bondi systems are related by the so-called BMS transformations,

$$
\begin{gathered}
u^{\prime}=K(\zeta, \bar{\zeta})(u-\gamma(\zeta, \bar{\zeta})), \\
\zeta^{\prime}=\frac{A \zeta+B}{C \zeta+D}
\end{gathered}
$$

where

$$
K(\zeta, \bar{\zeta})=\frac{1+\zeta \bar{\zeta}}{(A \zeta+B)(\bar{A} \bar{\zeta}+\bar{B})+(C \zeta+D)(\bar{C} \bar{\zeta}+\bar{D})}
$$

$\gamma(\zeta, \bar{\zeta})$ is an arbitrary real regular function of the angular variables, and $(A, B, C, D)$ are complex parameters satisfying $A D-B C=1$. The $\gamma$ freedom is known as the supertranslation.

In special relativity, rest frames are determined by those Cartesian inertial frames for which the momentum vector has only a timelike component different from zero or, in other words, those for which the generator of time translations of the frame is aligned with the total momentum.

At future null infinity this situation is complicated by the fact that, although there is a unique definition of momentum, there are several definitions of supermomenta. This means that there are several alternative nonequivalent definitions of rest frames at $\mathcal{I}^{+}$. Next, we review some possible definitions of supermomenta.

\section{Supermomenta}

Given an arbitrary section $\mathbf{S}$ of $\mathcal{I}^{+}$, one can choose, without loss of generality, a Bondi coordinate system $(u, \zeta, \bar{\zeta})$, such that $u=u_{0}=$ constant determines the section $\mathbf{S}$. Then, the different supermomenta on $\mathbf{S}$ can be expressed in terms of the corresponding integrands as

$$
P_{[X] l m}(\mathbf{S})=-\frac{1}{\sqrt{4 \pi}} \int_{\mathbf{S}} Y_{l m}(\zeta, \bar{\zeta}) \Psi_{[X]}\left(u=u_{0}, \zeta, \bar{\zeta}\right) d S^{2},
$$

where $d S^{2}$ is the surface element of the unit sphere on $\mathbf{S}$, $Y_{l m}$ are the spherical harmonics, and $[X]$ indicates the type of supermomentum. 
Among the different possibilities let us mention the following: the Geroch [9,22] supermomentum, with integrand

$$
\Psi_{[G]}=\Psi_{2}^{0}+\sigma_{0} \dot{\bar{\sigma}}_{0}+\frac{1}{2}\left(ð_{0}^{2} \bar{\sigma}_{0}-\bar{\jmath}_{0}^{2} \sigma_{0}\right)
$$

the Geroch-Winicour [8] supermomentum, with integrand [15]

$$
\Psi_{[G W]}=\Psi_{2}^{0}+\sigma_{0} \dot{\bar{\sigma}}_{0}-\overline{\mathrm{d}}_{0}^{2} \sigma_{0} ;
$$

and the supermomentum which we have used in the past [13] for defining nice sections, with integrand

$$
\Psi_{[M]}=\Psi_{2}^{0}+\sigma_{0} \dot{\bar{\sigma}}_{0}+ð_{0}^{2} \bar{\sigma}_{0} .
$$

In all these expressions, dots over quantities $f$ denote Bondi time derivatives, i.e. $\dot{f}=\frac{\partial f}{\partial u}$; $\Psi_{2}^{0}$ is the leading order part in the asymptotic expansion of the component,

$$
\Psi_{2}=C_{a b c d} l^{a} m^{b} \bar{m}^{c} n^{d},
$$

of the Weyl tensor $C_{a b c d}$, in an expansion in terms of powers of $1 / r$ around $\mathcal{I}^{+}$, i.e.

$$
\Psi_{2}=\frac{\Psi_{2}^{0}}{r^{3}}+\frac{\Psi_{2}^{1}}{r^{4}}+\cdots ;
$$

the scalar $\sigma_{0}$ is the leading order part in the asymptotic expansion of the shear,

$$
\sigma=m^{a} m^{b} \nabla_{b} l_{a} ;
$$

and we use the symbol $ð_{0}$ to denote the edth operator of the unit sphere.

All these supermomenta have the property that the first four components of the supermomentum, namely, the case $l=0$ and the three cases $l=1$, determine the Bondi energy-momentum vector. In other words,

$$
\left(P^{\mathrm{a}}\right)=\left(P_{00},-\frac{1}{\sqrt{6}}\left(P_{11}-P_{1,-1}\right), \frac{i}{\sqrt{6}}\left(P_{11}+P_{1,-1}\right), \frac{1}{\sqrt{3}} P_{10}\right),
$$

where $\mathrm{a}=0,1,2,3$.

The quantity $\Psi_{[M]}$ has some interesting properties: it is real, $\Psi_{[M]}=\bar{\Psi}_{[M]}$, and also $\dot{\Psi}_{[M]}=\dot{\sigma}_{0} \dot{\bar{\sigma}}_{0}$.

\section{Nice sections}

Nice sections provide a determination of cuts, S. Given an initial section $\mathbf{S}_{0}$, we can always think, without loss of generality, that there is a Bondi coordinate such that the section coincide with $u=0$. One can determine any other section, by the supertranslation $\gamma$ that takes one from $\mathbf{S}_{0}$, to
$\mathbf{S}$; which coincides with the section $u^{\prime}=u-\gamma=0$ in a new Bondi coordinate system.

At the section $\mathbf{S}$ we require the supermomentum $P_{[M] l m}(\mathbf{S})$ to have zero spatial components, providing us with a geometric notion of rest frame. In other words, only $P_{[M] 00}(\mathbf{S})$ is nonvanishing. This, in general, involves the need to make a Lorentz boost which keeps $\mathbf{S}$ fixed, but it aligns the generator of time translations with the total momentum.

It was shown in the past $[13,14]$ that this condition can be cast in the following equation:

$$
\mathrm{\partial}_{0}^{2} \overline{\mathrm{\gamma}}_{0}^{2} \gamma=\Psi_{[M]}(\gamma, \zeta, \bar{\zeta})+K^{3}(\gamma, \zeta, \bar{\zeta}) M(\gamma),
$$

where $\gamma$ is the supertranslation which determines the nice section, $K$ is the conformal boost factor [11], and $M$ is the mass at the section $\mathbf{S}$ given by

$$
M=\sqrt{P^{\mathrm{a}} P_{\mathrm{a}}} .
$$

Some expected physical properties of the nice section equation were proven in Refs. [14,15] as follows:

(i) There exists a four-parameter family of solutions of the nice section equation, for radiating spacetimes.

(ii) Having a nice section $S_{0}$, all other nice sections $S_{f}$ obtained from future timelike translations happen to be to the future of $S_{0}$.

(iii) If the spacetime is stationary, then the nice section equation reduces to the good cut equation [23]. We should emphasize that the good cut equation only admits solutions in the case of stationary spacetimes, whereas the nice section equation always has solutions.

Let us note that if we tried to do a similar construction of nice sections using, instead of the supermomentum $\Psi_{[M]}$, the Geroch supermomentum $\Psi_{[G]}$, then one would not be able to obtain equations that determine sections. This is due to the fact that under BMS transformations, the expression $\tilde{\partial}^{2} \tilde{\bar{\sigma}}_{0}$ transforms as

$$
\begin{aligned}
\tilde{\partial}^{2} \tilde{\bar{\sigma}}_{0}= & \frac{1}{K^{3}}\left(ð^{2} \bar{\sigma}_{0}-ð^{2} \bar{\partial}^{2} \gamma\right)+\frac{1}{K^{3}} \\
& \times\left[2 ð \gamma \partial \dot{\overline{\sigma_{0}}}+ð^{2} \gamma \dot{\overline{\sigma_{0}}}+(ð \gamma)^{2} \ddot{\bar{\sigma}}_{0}\right],
\end{aligned}
$$

and therefore the transformation rule for $\Psi_{[G]}$ is

$$
\tilde{\Psi}_{[G]}=\frac{1}{K^{3}}\left\{\Psi_{[G]}-\frac{1}{2}\left[2 ð \gamma \partial \dot{\overline{\sigma_{0}}}+ð^{2} \gamma \dot{\overline{\sigma_{0}}}+(ð \gamma)^{2} \ddot{\overline{\sigma_{0}}}+\text { c.c }\right]\right\} .
$$

In this way, in the case of a stationary spacetime, we would not have any equation for sections, since in such situations, $\Psi_{[G]}$ is supertranslation invariant; in particular, we would not recover the good cuts. Also, if we had used the $\Psi_{[G W]}$ supermomentum, it would not have the pleasant property of 
a positive-definite time derivative, which would complicate the discussion of its properties.

The nice section construction singles out precisely, in an intrinsic way, a Poincare structure from the infinite-dimensional BMS group. In particular, given a fixed observational point $p$ at $\mathcal{I}^{+}$, there is precisely a 3-degreesof-freedom set of spacelike translations which generate all the nice sections that contain $p$. In contrast, without this construction there is an infinite-dimensional family of general sections that contain $p$, one for each supertranslation. In particular, for stationary spacetimes, the nice sections requirement selects those whose shear is zero [11].

\section{THE KOMAR ANGULAR MOMENTUM IN AXIALLY SYMMETRIC SPACETIMES AT NULL INFINITY}

Before discussing the charge-integral approach to the notion of intrinsic angular momentum, let us review the definition of angular momentum through the Komar integral. Let $\left(M, g_{a b}\right)$ be an axially symmetric asymptotically flat spacetime. Therefore, it admits a Killing vector $v^{a}$ with closed orbits, and so it satisfies

$$
\nabla_{(b} v_{a)}=0 .
$$

Let $\mathbf{S}$ be a sphere; then the Komar integral [24] $K_{\mathbf{S}}(v)$ is defined by

$$
K_{\mathbf{S}}(v)=-\frac{1}{16 \pi} \int_{\mathbf{S}} \nabla^{a} v^{b} d S_{a b},
$$

where $d S_{a b}$ is the surface element of $\mathbf{S}$.

Let $\mathbf{S}^{\prime}$ be any other two-surface, and let $\Sigma$ be a hypersurface which has as boundaries both $\mathbf{S}$ and $\mathbf{S}^{\prime}$. Then the difference of the Komar integrals at $\mathbf{S}$ and $\mathbf{S}^{\prime}$ is given by

$$
K_{\mathbf{S}}(v)-K_{\mathbf{S}^{\prime}}(v)=\frac{1}{16 \pi} \int_{\Sigma} R_{b}^{a} v^{b} d S_{a},
$$

where $d S_{a}$ is the volume element of $\Sigma$ and $R_{b}^{a}$ is the Ricci tensor.

This means that in vacuum, the Komar integral is conserved. It is because of this property that these integrals are appreciated so much.

At future null infinity, a rotational Killing vector $v^{a}$ can be thought to be tangent to a sphere $\mathbf{S}$, and it can be expressed as

$$
v^{a}=-v_{\bar{m}} \hat{m}^{a}-v_{m} \overline{\hat{m}}^{a}=-\bar{\jmath}_{0} \overline{\tilde{a}} \hat{m}^{a}-\mathrm{\jmath}_{0} \tilde{a} \overline{\hat{m}}^{a},
$$

where $\hat{m}^{a}$ is the leading order part in the asymptotic expansion of the vector $m^{a}$ and we have used the fact that $v_{\bar{m}}$ and $v_{m}$ must be quantities of spin weight $s=-1$ and $s=1$, respectively; therefore, they can be expressed in terms of a spin-zero quantity $\tilde{a}$ through the edth operator.
After some computations, which are shown in the Appendix, taking $\tilde{a}=a$, the Komar angular momentum can be written as

$$
K_{\mathbf{S}}(v)=\frac{1}{8 \pi} \int_{\mathbf{S}} \bar{\partial}_{0} \overline{\tilde{a}}\left(\Psi_{1}^{0}+\sigma_{0} \varlimsup_{0} \bar{\sigma}_{0}\right) d S^{2}+\text { c.c. }
$$

\section{CHARGE INTEGRALS AT FUTURE NULL INFINITY}

It is convenient to approach the concept of physical quantities by the method of charge integrals. For example, in electromagnetism, the charge enclosed by a two-surface $\mathbf{S}$ is given by

$$
Q=-k \int_{\mathbf{S}}^{*} F,
$$

where $k$ is a constant which depends on the units.

Let us remark that the electromagnetic tensor $F$ can be understood as the curvature of the connection $A$, identified with the potential vector.

In a similar way, a charge integral can be obtained from the Riemann curvature tensor.

Given a two-sphere $\mathbf{S}$, we will work with the charge integral of the Riemann tensor:

$$
Q_{\mathrm{s}}=\int_{\mathbf{S}} C
$$

where the two-form $C_{a b}$ is given by

$$
C_{a b} \equiv R_{a b}^{* c d} w_{c d},
$$

with $R_{a b}^{* c d}=\frac{1}{2} R_{a b e f} \epsilon^{e f c d}$ the right dual of the Riemann tensor and $w_{a b}$ a two-form which will be determined next. For motivations of these types of charge integrals, see $[4,16,25]$.

At this point, one might wonder why we use the right dual of the Riemann tensor instead of the left dual; the answer is that by using the right dual, we can directly relate the exterior derivative of the form $C_{a b}$ with the Einstein tensor as we show below.

Let $\Sigma$ be a spacelike hypersurface in the interior of the spacetime which asymptotically reaches future null infinity in such a way that in the conformally completed spacetime, $\Sigma$ can be extended to $\mathcal{I}^{+}$with boundary $\mathbf{S}$. Then, by using Stokes' theorem the charge integral on $\mathbf{S}$ can be expressed as an integral on $\Sigma$, namely,

$$
Q_{\mathbf{S}}=\int_{\mathbf{S}} C=\int_{\Sigma} d C
$$

The exterior derivative of $C$ can be expressed [4] by

$$
d C_{a b c}=\frac{1}{3} \epsilon_{a b c d}{ }^{*} R^{* \operatorname{def} g} \nabla_{e} w_{f g} .
$$


As it was said before, an important property of the double dual of the Riemann tensor is that its trace gives the Einstein tensor, namely,

$$
{ }^{*} R_{a b c d}^{*} g^{b d}=G_{a c}=R_{a c}-\frac{1}{2} g_{a c} R .
$$

Therefore, the previous equation can be written as

$$
d C_{a b c}=\frac{1}{3} \epsilon_{a b c d}\left(-\frac{2}{3} G^{d g} v_{g}+{ }^{*} R^{* \operatorname{defg} T_{e f g}}\right),
$$

where $T_{a b c}$ is the traceless part of $\nabla_{a} w_{b c}$ and $v_{c}$ its trace; that is,

$$
\nabla_{a} w_{b c}=T_{a b c}+\frac{1}{3} g_{a b} v_{c}-\frac{1}{3} g_{a c} v_{b},
$$

with

$$
\nabla_{a} w^{a b}=v^{b} .
$$

Let us study, for a moment, this expression from the point of view of linearized gravity. Suppose that the metric is expressed as $g=\eta+h$, in terms of a flat background metric $\eta$. Then, from Eqs. (28) and (30) one observes that if the vector $v^{a}$ is a Killing vector of the metric $\eta_{a b}$ and if $T_{a b c}$ is $O(h)$, then the charge integral will give the conserved quantities in the context of linearized gravity. It is clear that one can always find such a $w$. Then, this analysis ensures that the charge integrals admit the appropriate physical interpretations in the linearized gravity regime and, in particular, that they do not suffer from the factor of 2 anomaly [7].

Another property of the double dual of the Riemann tensor is the one associated with the Bianchi identities, namely, ${ }^{*} R^{* d[e f g]}=0$, from which one can prove [11] the relation

$$
{ }^{*} R^{* \operatorname{defg}} T_{e f g}=\frac{2}{3} * R^{* \operatorname{defg}}\left(T_{(e f) g}-T_{(e g) f}\right) .
$$

This expression can be written in a simpler form using spinorial notation. $T_{a b c}$ can be expressed [11] as

$$
\frac{2}{3}\left(T_{(e f) g}-T_{(e g) f}\right)=\nabla_{E^{\prime}\left(E^{\left.W_{F G}\right)}\right.} \epsilon_{F^{\prime} G^{\prime}}+\text { c.c. },
$$

where c.c. means complex conjugate, and we have made the standard abuse of notation, identifying the vectorial abstract indices with spinorial abstract indices using the rule $e=E E^{\prime}$.

From these considerations, the most natural conditions on $w$ are to stay as close as possible to the following conditions:

$$
-\nabla_{A} B^{\prime} w^{A B}+\text { c.c. }=v^{B B^{\prime}}
$$

and

$$
\nabla_{E^{\prime}\left(E^{\left.w_{F G}\right)}\right.}=0,
$$

where the vector $v^{B B^{\prime}}$ is a generator of asymptotic symmetries.

In general, an asymptotic symmetry $v^{a}$ can be expressed by its components in terms of a null tetrad frame,

$$
v^{a}=v_{n} \ell^{a}-v_{\bar{m}} m^{a}-v_{m} \bar{m}^{a}+v_{\ell} n^{a} .
$$

Since the asymptotic symmetries are tangent to $\mathcal{I}^{+}$, the tetrad components have the following behavior:

$$
\begin{aligned}
& v_{n}=r v_{n}^{0}+v_{n}^{1}+O\left(\frac{1}{r}\right), \\
& v_{m}=r v_{m}^{0}+v_{m}^{1}+O\left(\frac{1}{r}\right), \\
& v_{\ell}=v_{\ell}^{0}+\frac{v_{\ell}^{1}}{r}+O\left(\frac{1}{r^{2}}\right) .
\end{aligned}
$$

The leading order part in the asymptotic expansion in a Bondi system of an asymptotic symmetry is given by

$$
\begin{aligned}
& v_{m}^{0}=ð_{0} a, \\
& v_{n}^{0}=\frac{1}{2}\left(\partial_{0} v^{0}{ }_{\bar{m}}+\overline{\mathrm{\partial}}_{0} v^{0}{ }_{m}\right)=\frac{1}{2}{\mathrm{\partial}_{0}}_{\overline{\mathrm{d}}_{0}}(a+\bar{a}), \\
& v_{l}^{0}=\chi(\zeta, \bar{\zeta})-u \frac{1}{2}{\partial_{0}}_{\bar{\partial}_{0}}(a+\bar{a}),
\end{aligned}
$$

where $\chi$ and $a$ are functions on the sphere with spin weight 0 , satisfying $\chi=\bar{\chi}, \dot{a}=0$ and $\mathrm{\partial}_{0}^{2} a=0$.

Relation (33) at $\mathcal{I}^{+}$can be expressed in terms of the spinorial components of a regular dyad,

$$
w^{A B}=w_{0} \hat{l}^{A} \hat{l}^{B}-w_{1}\left(\hat{o}^{A} \hat{\imath}^{B}+\hat{\imath}^{A} \hat{o}^{B}\right)+w_{2} \hat{\imath}^{A} \hat{l}^{B},
$$

by

$$
\begin{gathered}
w_{2}=-\frac{1}{3} v_{\bar{m}}^{0}, \\
w_{1}+\bar{w}_{1}=-\frac{1}{3} v_{\ell}^{0}, \\
\dot{w}_{1}+\dot{\bar{w}}_{1}=-\frac{1}{2}\left({ }_{0} w_{2}+\bar{\partial}_{0} \bar{w}_{2}\right),
\end{gathered}
$$

while condition (34) at $\mathcal{I}^{+}$becomes

$$
\begin{gathered}
\overline{\mathrm{d}}_{0} w_{2}=0, \\
\dot{w}_{2}=0,
\end{gathered}
$$




$$
\begin{gathered}
\mathrm{\jmath}_{0} w_{0}=-2 \sigma_{0} w_{1}, \\
\dot{w}_{1}=-\frac{1}{2} \varlimsup_{0} w_{2}, \\
\frac{1}{2} \dot{w}_{0}+\jmath_{0} w_{1}+\sigma_{0} w_{2}=0 .
\end{gathered}
$$

It was shown in Ref. [11] that the charge integral at future null infinity can be expressed as

$Q_{\mathbf{S}}(w)=4 \int\left[-w_{2} \Psi_{1}^{0}+2 w_{1}\left(\Psi_{2}^{0}+\sigma_{0} \dot{\bar{\sigma}}_{0}\right)\right] d S^{2}+$ c.c.,

where $\Psi_{1}^{0}$ is the leading order part in the asymptotic expansion of the tetrad component of the Weyl tensor,

$$
\Psi_{1}=C_{a b c d} l^{a} n^{b} m^{c} l^{d} .
$$

\section{CHARGE INTEGRALS IN STATIONARY SPACETIMES AND PHYSICAL QUANTITIES}

For the case of stationary spacetimes one can solve the set of Eqs. (43)-(50) with the solution

$$
\begin{aligned}
& w_{2}=-\frac{1}{3} \bar{\partial}_{0} \bar{a}, \\
& w_{1}=w_{1}^{00}\left(\zeta, \bar{\zeta}, \sigma_{0}\right)+\frac{1}{6} u \mathrm{\jmath}_{0} \bar{\jmath}_{0} \bar{a}, \\
& w_{0}=w_{0}^{00}\left(\zeta, \bar{\zeta}, \sigma_{0}\right)+u\left(-2 ð_{0} w_{1}^{00}+\frac{2}{3} \sigma_{0} \overline{\mathrm{d}}_{0} \bar{a}\right) \\
& -\frac{1}{6} u^{2}{\mho_{0}^{2}}_{0} \bar{\partial}_{0} \bar{a},
\end{aligned}
$$

where $\bar{ळ}_{0}^{2} \bar{a}=0$, and $w_{1}^{00}$ and $w_{0}^{00}$ are spin weight 0 and 1 functions, respectively, that solve the equations

$$
ð_{0}^{2} w_{1}^{00}=\frac{1}{3} ð_{0} \sigma_{0} \overline{\partial_{0}} \bar{a}+\frac{1}{2} \sigma_{0} ð_{0} \bar{\partial}_{0} \bar{a}=-ð_{0} \sigma_{0} w_{2}-\frac{3}{2} \sigma_{0} ð_{0} w_{2}
$$

and

$$
ð_{0} w_{0}^{00}=-2 \sigma_{0} w_{1}^{00} .
$$

Let us note that if one uses the potential $\delta$ of the shear satisfying

$$
\sigma_{0}=\mathrm{\partial}_{0}^{2} \delta
$$

then the component $w_{1}$ can be expressed by

$$
w_{1}=b+\frac{1}{3}{\mathrm{\partial}_{0}}_{\mathrm{\partial}_{0}} \bar{a}+\frac{1}{6}(u-\delta) \mathrm{\partial}_{0} \overline{\mathrm{\partial}}_{0} \bar{a},
$$

where the spin 0 quantity $b$ satisfies $\dot{b}=0$ and $ð_{0}^{2} b=0$.
This procedure provides us with a two-form $w_{A B}^{0}$ given by Eqs. (53)-(55), with the functional dependence on $u=\gamma$ given by

$$
w_{A B}^{0}(\gamma)=w_{A B}^{0}\left(u=\gamma, \zeta, \bar{\zeta} ; \sigma_{0}(\zeta, \bar{\zeta}), a, b\right),
$$

where we stress the dependence on $\sigma_{0}$.

Let us observe that $a$ involves six real constants associated with the Lorentz rotations and that, since in this case $\Psi_{2}^{0}$ is a real quantity, $b$ contributes to the charge integral $Q_{\mathbf{S}}(w)$ with four other real constants associated with translations.

The first term in the integrand of Eq. (51) includes the Weyl component $\Psi_{1}$ which is known to describe the angular momentum in the Kerr geometry. In the second term we recognize the component $\Psi_{2}^{0}$ which determines the supermomentum $\Psi_{[M]}$ for this particular stationary case.

Let us recall that in special relativity, angular momentum and intrinsic angular momentum are related by expressions of the form $J^{\mathrm{ab}}=S^{\mathrm{ab}}+R^{\mathrm{a}} P^{\mathrm{b}}-P^{\mathrm{a}} R^{\mathrm{b}}$. Then, given a rest reference frame in Minkowski spacetime, one needs to use the spacelike translation freedom $\left(R^{\mathrm{b}}\right)$ in order to single out the center-of-mass reference frame. In the center-of-mass frame one has $J^{\mathrm{ab}}=S^{\mathrm{ab}}$. Also, since the intrinsic angular momentum satisfies $S^{\mathrm{ab}} P_{\mathrm{b}}=0$ (frequently referred to as the Dixon condition [26]), one can characterize the centerof-mass frame as that rest frame for which $J^{0 i}=0$. It can be seen that the condition we need to impose on the section $\mathbf{S}$ in the charge-integral case is that it must be the nice section satisfying

$$
Q_{\mathbf{S}}(a)=0 \quad \text { for all } a=\bar{a},
$$

where it is understood that one takes $b=0$ in this equation. The quantity $a$ is, in principle, complex, so this condition makes use of precisely 3 degrees of freedom, which are associated with spacelike translations.

This is the appropriate condition which leaves a onedimensional family of nice sections $\mathbf{S}$ that can legitimately be called center-of-mass frames. In particular, we can see that the other center-of-mass frames are generated by time translations from an original one in the nice section construction. Using these frames $\mathbf{S}_{\mathrm{cm}}$, the intrinsic angular momentum $\mathrm{s}$ is defined by

$$
\mathrm{S}=\frac{3}{32 \pi} Q_{\mathbf{s}_{\mathrm{cm}}}(w),
$$

where to determine $w$ one chooses $a=-\bar{a}$ and $b=0$. Note also that the same charge integral can be used to calculate the Bondi momentum $\mathrm{p}$ given by

$$
\mathrm{p}=\frac{3}{32 \pi} Q_{\mathbf{s}_{\mathrm{cm}}}(w),
$$

where in this case one takes $a=0$ and $b \neq 0$. 
The previous prescription singles out the center-of-mass frame for stationary spacetimes and a Poincaré subgroup of BMS generators.

At this point, it is probably worthwhile to mention that some authors have considered an alternative to the Dixon condition, known as the Mathisson condition [27], which requires $J^{a b} U_{b}=0$, with $U^{b}$ the four-velocity vector of the worldline associated with the center of mass. But this condition has the difficulty that it does not prescribe a unique worldline; in fact, the solution depends on the choice of an initial $U^{b}$, which gives rise to helical motion for free spinning particles, instead of the geodesic motion. Some authors have given physical meaning to these curves [28], and recent articles discuss the range of validity (see, for example, [29]). We do not make use of this alternative condition because we require a definition of center of mass and intrinsic angular momentum to be constructed from intrinsic physical quantities, avoiding arbitrary choices.

\section{INTRINSIC ANGULAR MOMENTUM FOR RADIATING SPACETIMES WHICH AGREES WITH THE KOMAR INTEGRAL}

We show in detail how to obtain the intrinsic angular momentum for the general radiating case.

As before, we define the rest frame sections as those for which $P_{[M] l m}(\mathbf{S})=0, \forall l \geq 1$.

Let us consider a point along a particular generator of $\mathcal{I}^{+}$, denoted by $p(\tau)$, with $\tau$ a monotonically increasing time parameter. The set of nice sections form a four-parameter $(T, \vec{R})$ family that we now label $\mathbf{S}_{(T, \vec{R})}$, where $(T, \vec{R})$ can be identified with a translation among nice sections. Then, for a given fixed $\tau$, one has a three-parameter family of nice sections $\mathbf{S}_{(T, \vec{R})}$ which contains the point $p(\tau)$.

Given one of these nice sections $\mathbf{S}_{(T, \vec{R})}$, we can always identify it with the condition $u=\gamma_{\mathbf{S}}$, where $\gamma_{\mathbf{S}}(\zeta, \bar{\zeta})$ is the supertranslation that defines the corresponding section.

At this point it is important to emphasize thatEqs. (46)-(50), in general, do not have solutions in a radiating spacetime. In spite of that, we can propose a prescription which defines a two-form suitable for our construction.

On $\mathbf{S}_{(T, \vec{R})}$ we define the two-form $w_{\mathbf{S}_{(T, \vec{R})}}$ as the solution of the stationary problem [(53)-(55)] where the radiation data are taken as $\sigma_{\mathbf{S}_{(T, \vec{R})}}(u, \zeta, \bar{\zeta})=\sigma_{0}\left(\gamma_{\mathbf{S}}, \zeta, \bar{\zeta}\right)$.

Then, using the identity

$$
\int_{\mathbf{S}}\left(w_{1} ð_{0}^{2}{\overline{\sigma_{0}}}_{)} d S^{2}=\int_{\mathbf{S}}\left(\sigma_{0}{\partial_{0}}_{\sigma_{0}}+\frac{1}{2} ð_{0}\left(\sigma_{0} \bar{\sigma}_{0}\right)\right) w_{2} d S^{2},\right.
$$

we see that the charge integral (51) can be expressed more generally as

$$
\begin{aligned}
Q_{\mathbf{S}}(w)= & 4 \int_{\mathbf{S}}\left\{-w_{2}\left[\Psi_{1}^{0}-\alpha\left(\sigma_{0}{\mathrm{\partial}_{0}}_{\overline{\sigma_{0}}}+\frac{1}{2}{\mathrm{\partial}_{0}}\left(\sigma_{0} \overline{\sigma_{0}}\right)\right)\right]\right. \\
& \left.+2 w_{1}\left[\Psi_{2}^{0}+\sigma_{0} \dot{\overline{\sigma_{0}}}-\frac{\alpha}{2} ð_{0}^{2} \overline{\sigma_{0}}\right]\right\}+ \text { c.c. },
\end{aligned}
$$

with $\alpha$ a constant. Let us note that using the potential $w_{2}$, the Komar angular momentum (22) can be written as

$$
K_{\mathbf{S}}(v)=\frac{3}{8 \pi} \int_{\mathbf{S}}-w_{2}\left(\Psi_{1}^{0}+\sigma_{0}{\partial_{0}}_{\sigma_{0}}\right) d S^{2}+\text { c.c. }
$$

Therefore, in order to recover the Komar expression for the angular momentum, in the case of axisymmetric spacetimes, we must set $\alpha=-1$.

In this way, the expression for the charge integral (that it can be used to calculate angular momentum, momentum or supermomentum) is

$$
\begin{aligned}
Q_{\mathbf{S}}(w)= & 4 \int_{\mathbf{S}}\left\{-w_{2}\left[\Psi_{1}^{0}+\sigma_{0}{\mathrm{\partial}_{0}}_{\overline{\sigma_{0}}}+\frac{1}{2}{\partial_{0}}_{0}\left(\sigma_{0} \bar{\sigma}_{0}\right)\right]\right. \\
& \left.+2 w_{1}\left(\Psi_{[M]}-\frac{1}{2}{\partial_{0}^{2}}_{\overline{\sigma_{0}}}\right)\right\}+ \text { c.c. }
\end{aligned}
$$

It is interesting to note that the first integrand factor coincides with that obtained by Winicour [7] (except for relative signs, due to differences in conventions).

Let us note, then, that the first term takes the form of the Komar angular momentum since one can check that when $3 w_{2}$ is the component of a rotational Killing vector, the term $\mathrm{\partial}_{0}\left(\sigma_{0} \bar{\sigma}_{0}\right)$ does not contribute. Also, let us observe that in the case of a stationary spacetime, it reduces to the result of the previous section since the center-of-mass sections coincide with the sections with $\sigma_{0}=0$.

In addition, in order to compute the intrinsic angular momentum, for each choice of $a$ we take $b(a)$. which satisfies

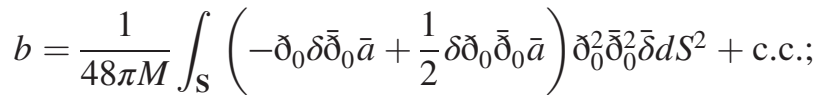

where $\delta$ is the complex potential for the shear defined by

$$
\sigma=ð_{0}^{2} \delta .
$$

This choice of $b$ is made so that the second term in (67) does not contribute; thus, the complete charge integral coincides with the Komar integral.

Similarly to how it was done before, in order to single out the center-of-mass section $\mathbf{S}_{\mathrm{cm}}$ from the three-parameter family of nice sections which contain the point $p(\tau)$, we demand 


$$
Q_{\mathbf{S}_{\mathrm{cm}}}(a)=0 \text { for all } a=\bar{a} .
$$

Using these center-of-mass frames $\mathbf{S}_{\mathrm{cm}}$, the intrinsic angular momentum $\mathbf{S}$ is defined through

$$
\mathbf{s}=\frac{3}{32 \pi} Q_{\mathbf{S}_{\mathrm{cm}}}(w),
$$

where, as before, in order to pick up the intrinsic angular momentum, one takes $a=-\bar{a}$.

In this approach, the observer (us) is located at future null infinity, confined to a particular generator. Then, for each retarded time, our construction singles out a unique center-of-mass section, where the calculation of the intrinsic angular momentum is carried out. Thus, if one wants to compare the spin at two different times, one has to repeat the construction of the center-of-mass section at the second reference time. Let us also note that since between two center-of-mass sections we have at our disposal the oneparameter family of center-of-mass sections among them, we also have the two-form $C_{a b}$, on this region, and therefore, the difference of the spins can be expressed as a flux law, using Stokes' theorem.

\section{FINAL COMMENTS}

We have shown in detail how the problem of supertranslations, in defining angular momentum, can be circumvented with the help of the so-called nice sections and the charge integrals.

The comparison of the present approach based on charge integrals of the Riemann tensor with traditional approaches has been done in Ref. [11]. Among the recent contributions on the subject, we comment on the ingenious work of Ref. [30] based in Dirac eigenspinors. The application of the so-called spectral angular momentum to our example [18] of a supertranslated boosted section in Schwarzschild spacetime captures the nonzero value of the orbital angular momentum. In contrast, in our approach, the intrinsic angular momentum for any center-of-mass section gives the expected zero value.

As was mentioned in the Introduction, we emphasize again that our work tackles the definition of intrinsic angular momentum as opposed to just total angular momentum as discussed by most other works. It is the notion of intrinsic angular momentum that is relevant to the study of astrophysical systems and, in particular, to the problem of balance of gravitational radiation.

Another point that is worthwhile to note is that the definition presented here satisfies the property that in a spacetime with three stages-an axisymmetric one, a nonaxisymmetric one, and a third with a different Killing symmetry-it gives the expected values at the first and third stages.

In summary, we have presented a definition of intrinsic angular momentum which is free from supertranslation dependence. It can be applicable to a general radiating spacetime, and it agrees, for the case of axial symmetry, with the Komar integral.

To our knowledge, this is the only definition of intrinsic angular momentum with these properties.

\section{ACKNOWLEDGMENTS}

We are grateful to László Szabados for deep illuminating discussions and for kind hospitality at the Wigner Research Centre for Physics, KFKI, Budapest. We would also like to acknowledge the valuable comments and criticism from anonymous referees, which helped us to considerably improve the presentation of our work. We acknowledge financial support from CONICET and SeCyT-UNC.

\section{APPENDIX: THE KOMAR EXPRESSION FOR ANGULAR MOMENTUM}

In an axisymmetric spacetime there exists a Killing vector field $v^{a}$ associated with the axial symmetry. If the spacetime is also asymptotically flat, then the Komar definition of angular momentum is

$$
K_{\mathbf{S}}(v)=-\frac{1}{16 \pi} \int_{\mathbf{S}} \nabla^{b} v^{a} d S_{a b},
$$

where $d S_{a b}$ is the surface element of a two-sphere $\mathbf{S}$ defined as a cut of $\mathcal{I}^{+}$. An operational way to explicitly write this integral is to consider a Bondi system $(u, \zeta, \bar{\zeta})$ such that we extend $\mathbf{S}$ to the interior of the spacetime along null geodesics with tangent vector $l^{a}$ such that they are orthogonal to $\mathbf{S}$ at $\mathcal{I}^{+}$. This construction generates a null surface given by $u=$ constant, with $l_{a}=(d u)_{a}$. If we also define an affine parameter $r$ along the null geodesics $l^{a}$, then the two-surfaces $S_{u, r}$ defined by $r=$ constant on $u=$ constant will be two-spheres. We can then complete $l^{a}$ to a null tetrad $\left\{l^{a}, n^{a}, m^{a}, \bar{m}^{a}\right\}$, by making $m^{a}$ and $\bar{m}^{a}$ tangent to the two-spheres $S_{u, r}$. Then, the Komar angular momentum (A1) can be reexpressed as

$$
K_{\mathbf{S}}(v)=-\frac{1}{8 \pi} \lim _{r \rightarrow \infty} \int_{\mathbf{S}} \nabla^{b} v^{a} l_{[a} n_{b]} d \tilde{S}^{2},
$$

where we used $d S_{a b}=2 l_{[a} n_{b]} d \tilde{S}^{2}$, with $d \tilde{S}^{2}$ the surfacearea element of the spheres $S_{u, r}$. Now, the Killing vector $v^{a}$ is, by construction, tangent to the two-spheres $S_{u, r}$; then, it must be expressed as

$$
v^{a}=-v_{\bar{m}} m^{a}-v_{m} \bar{m}^{a}=-\bar{\jmath} \overline{\tilde{a}} m^{a}-ð \tilde{a} \bar{m}^{a},
$$

where in the last equality we used the fact that $v_{\bar{m}}$ and $v_{m}$ are quantities of spin weight $s=-1$ and $s=1$, respectively, and therefore can be written in terms of a spin-zero quantity $\tilde{a}$ through the edth operator. Then, by projecting the Killing equation $\nabla_{(a} v_{b)}=0$ in the direction of $m^{a} \bar{m}^{b}$, we get 


$$
\text { ðठ( }(\tilde{a}+\overline{\tilde{a}})=0 .
$$

This equation implies that $\tilde{a}+\overline{\tilde{a}}=$ constant; therefore, without loss of generality we can take $\tilde{a}+\overline{\tilde{a}}=0$. On the other hand, the integrand of (A2) reads

$$
\begin{aligned}
\nabla^{b} v^{a} l_{[a} n_{b]} & =-\nabla^{b}\left(\bar{\partial} \overline{\tilde{a}} m^{a}+ð \tilde{a} \bar{m}^{a}\right) l_{[a} n_{b]} \\
& =ð \tilde{a} \bar{\tau}+\bar{\jmath} \overline{\tilde{a}} \tau,
\end{aligned}
$$

with

$$
\tau=m^{a} n^{b} \nabla_{b} l^{a} .
$$

This means that in order to compute the angular momentum defined in (A2), we need to know the $O\left(r^{-2}\right)$ of $\partial \overline{\tilde{a}} \bar{\tau}$. It can be shown, from the Killing equations, that the term $\bar{\partial} \overline{\tilde{a}}$ has an asymptotic expansion as

$$
\bar{\delta} \overline{\tilde{a}}=v_{\bar{m}}^{0} r+v_{\bar{m}}^{1}+O\left(r^{-1}\right),
$$

with $v_{\bar{m}}^{1}$ and $v_{\bar{m}}^{0}$ functions of $(u, \zeta, \bar{\zeta})$. From the integration of the Killing equation

$$
\mathrm{P} v_{m}=-\sigma v_{\bar{m}}-\bar{\rho} v_{m},
$$

where $\mathrm{P}$, is the thorn operator in the GHP [17] notation, and taking into account the expansion in powers of $r$ of $\rho$ and $\sigma$,

$$
\begin{gathered}
\rho=-\frac{1}{r}+O\left(r^{-3}\right), \\
\sigma=\frac{\sigma_{0}}{r^{2}}+O\left(r^{-4}\right),
\end{gathered}
$$

it also follows that

$$
v_{\bar{m}}^{1}=\bar{\sigma}_{0} v_{m}^{0} .
$$

Let us also note that in terms of a regular tetrad, the Killing vector on $\mathcal{I}^{+}$is given by

$$
\left.v^{a}\right|_{\mathcal{I}^{+}}=-v_{\bar{m}}^{0} \hat{m}^{a}-v_{m}^{0} \hat{\bar{m}}^{a} .
$$

On the other hand, because $v_{\bar{m}}^{0}$ is a quantity of spin weight $s=-1$, it can be expressed in terms of a spin-weight 0 quantity $\bar{a}$ as

$$
v_{\bar{m}}^{0}=\bar{\jmath}_{0} \bar{a} .
$$

In the same way, it can be shown that $\tau$ has an asymptotic expansion as

$$
\tau=\bar{ळ}_{0} \sigma_{0} r^{-2}-\left(\Psi_{1}^{0}+2 \sigma_{0} \mathrm{\partial}_{0} \bar{\sigma}_{0}\right) r^{-3}+O\left(r^{-4}\right) .
$$

By expanding the product $\bar{\jmath} \overline{\tilde{a}} \tau$ up to order $O\left(r^{-2}\right)$, we obtain

$$
\begin{aligned}
\bar{\partial} \overline{\tilde{a}} \tau= & \overline{\mathrm{\partial}}_{0} \sigma_{0} v_{\bar{m}}^{0} r^{-1}+\left\{-\left[\Psi_{1}^{0}+2 \sigma_{0} \mathrm{\partial}_{0} \bar{\sigma}_{0}\right] v_{\bar{m}}^{0}+\bar{\sigma}_{0} \bar{\partial}_{0} \sigma_{0} v_{m}^{0}\right\} r^{-2} \\
& +O\left(r^{-3}\right) .
\end{aligned}
$$

Let us note from the first line of the previous equation, that not only does the leading order of the Killing vector make a contribution to the integral (given by $v_{\bar{m}}^{0}$ ) but it also contributes the term given by $v_{\bar{m}}^{1}$. Let us also note that the first term of this expression can be written as

$$
\begin{aligned}
& \overline{\mathrm{d}}_{0} \sigma_{0} v_{\bar{m}}^{0} r^{-1}=-\overline{\mathrm{d}}_{0} \sigma_{0} \overline{\mathrm{d}}_{0} \bar{a} r^{-1} \\
& =-\left[\overline{\mathrm{d}}_{0}\left(\sigma_{0} \overline{\mathrm{d}}_{0} \bar{a}\right)-\sigma_{0} \overline{\mathrm{\partial}}_{0}^{2} \bar{a}\right] r^{-1} \\
& =-\overline{\mathrm{d}}_{0}\left(\sigma_{0} \overline{\mathrm{d}}_{0} \bar{a}\right) r^{-1} \text {, }
\end{aligned}
$$

where we used the fact that $\bar{\delta}_{0}^{2} \bar{a}=0$, which follows from projecting the Killing equation $\nabla_{(a} v_{b)}=0$ in the direction of $m^{a} m^{b}$. Therefore, by adding its complex conjugate we obtain

$$
\begin{aligned}
ð \tilde{a} \bar{\tau}+\bar{\partial} \overline{\tilde{a}} \tau= & -\left[\overline{\mathrm{\partial}}_{0}\left(\sigma_{0} \overline{\mathrm{\partial}}_{0} \bar{a}\right)+\mathrm{\partial}_{0}\left(\bar{\sigma}_{0} \mathrm{\partial}_{0} a\right)\right] r^{-1} \\
& +\left[-\left(\Psi_{1}^{0}+\sigma_{0} \mathrm{\partial}_{0} \bar{\sigma}_{0}\right) \bar{\jmath}_{0} \bar{a}+\text { c.c }\right] r^{-2} \\
& +O\left(r^{-3}\right) .
\end{aligned}
$$

Then, the angular momentum reads

$$
K_{\mathbf{S}}(v)=\frac{1}{8 \pi} \int_{\mathbf{S}}\left(\Psi_{1}^{0}+\sigma_{0}{\partial_{0}}_{\bar{\sigma}_{0}}\right) \overline{\mathrm{d}}_{0} \bar{a} d S^{2}+\text { c.c. }
$$

with $d S^{2}$ the surface element of a unit two-sphere. This expression can be written in terms of

$$
w_{2}=-\frac{1}{3} \bar{\partial}_{0} \bar{a}
$$

as

$$
K_{\mathbf{S}}(v)=-\frac{3}{8 \pi} \int_{\mathbf{S}} w_{2}\left(\Psi_{1}^{0}+\sigma_{0}{\mathrm{\partial}_{0}}_{\bar{\sigma}_{0}}\right) d S^{2}+\text { c.c. }
$$


[1] H. Bondi, M. G. J. van der Burg, and A. Metzner, Proc. R. Soc. A 269, 21 (1962).

[2] R. Sachs, Phys. Rev. 128, 2851 (1962).

[3] R. K. Sachs, Proc. R. Soc. A 270, 103 (1962).

[4] O. M. Moreschi, Classical Quantum Gravity 3, 503 (1986).

[5] B. Bramson, Proc. R. Soc. A 341, 463 (1975).

[6] C. Prior, Proc. R. Soc. A 354, 379 (1977).

[7] J. Winicour, Angular Momentum in General Relativity, in “General Relativity and Gravitation," edited by A. Held (Plenum Press, New York, 1980), Vol. 2, pp. 71-96.

[8] R. Geroch and J. Winicour, J. Math. Phys. (N.Y.) 22, 803 (1981).

[9] T. Dray and M. Streubel, Classical Quantum Gravity 1, 15 (1984).

[10] L. B. Szabados, Living Rev. Relativity 7, 1 (2004), http:// relativity.livingreviews.org/Articles/lrr-2004-4.

[11] O. M. Moreschi, Classical Quantum Gravity 21, 5409 (2004).

[12] C. N. Kozameh and G. D. Quiroga, Classical Quantum Gravity 29, 235006 (2012).

[13] O. M. Moreschi, Classical Quantum Gravity 5, 423 (1988).

[14] O. M. Moreschi and S. Dain, J. Math. Phys. (N.Y.) 39, 6631 (1998).

[15] S. Dain and O. M. Moreschi, Classical Quantum Gravity 17, 3663 (2000).
[16] R. Penrose, Proc. R. Soc. A 381, 53 (1982).

[17] R. Geroch, A. Held, and R. Penrose, J. Math. Phys. (N.Y.) 14, 874 (1973).

[18] E. Gallo, L. Lehner, and O. M. Moreschi, Classical Quantum Gravity 26, 048002 (2009).

[19] C. O. Lousto and Y. Zlochower, Phys. Rev. D 76, 041502(R) (2007).

[20] R. Penrose, Phys. Rev. Lett. 10, 66 (1963).

[21] O. M. Moreschi, Classical Quantum Gravity 4, 1063 (1987).

[22] R. Geroch, in Asymptotic Structure of Space-time, edited by F. P. Esposito and L. Witten (Plenum Press, New York, 1977).

[23] E. T. Newman and R. Penrose, J. Math. Phys. (N.Y.) 7, 863 (1966).

[24] A. Komar, Phys. Rev. 113, 934 (1959).

[25] J. Goldberg, Phys. Rev. D 41, 410 (1990).

[26] W. Dixon, Proc. R. Soc. A 314, 499 (1970).

[27] M. Mathisson, Gen. Relativ. Gravit. 42, 1011 (2010); Acta Phys. Pol. 6, 163 (1937).

[28] C. Möller, Ann. Inst. Henri Poincaré 11, 251 (1949).

[29] L. F. O. Costa, C. A. Herdeiro, J. Natario, and M. Zilhao, Phys. Rev. D 85, 024001 (2012).

[30] L. B. Szabados, Classical Quantum Gravity 25, 025007 (2008). 